\title{
Phase Behavior of Symmetric Ternary Block Copolymer-Homopolymer Blends in Thin Films and on Chemically Patterned Surfaces
}

\author{
Mark P. Stoykovich, ${ }^{1}$ Erik W. Edwards, ${ }^{1}$ Harun H. Solak, ${ }^{2}$ and Paul F. Nealey ${ }^{1, *}$ \\ ${ }^{1}$ Department of Chemical and Biological Engineering and Center for Nanotechnology, University of Wisconsin, \\ Madison, Wisconsin 53706, USA \\ ${ }^{2}$ Laboratory for Micro- and Nanotechnology, Paul Scherrer Institute, Villigen CH-5232, Switzerland
} (Received 18 November 2005; revised manuscript received 21 July 2006; published 6 October 2006)

The phase diagram of symmetric ternary blends of diblock copolymers and homopolymers in thin films was determined as a function of increasing volume fraction of homopolymer $\left(\phi_{H}\right)$ and was similar to that for these materials in the bulk. Blends with compositions in the lamellar region of the diagram $\left(\phi_{H} \leq 0.4\right)$ could be directed to assemble into ordered lamellar arrays on chemically striped surfaces if the characteristic blend dimension $\left(L_{B}\right)$ and the period of the stripes $\left(L_{S}\right)$ were commensurate such that $L_{S}=$ $L_{B} \pm 0.10 L_{B}$. Blends with compositions in the microemulsion region of the diagram $\left(\phi_{H} \approx 0.6\right)$ assembled into defect-free lamellar phases on patterned surfaces with $L_{S} \geq L_{B}$, but formed coexisting lamellar (with period $L_{S}$ ) and homopolymer-rich phases when $L_{S}<L_{B}$.

DOI: 10.1103/PhysRevLett.97.147802

Blends of block copolymers and their corresponding homopolymers self-assemble into ordered phases with controllable shapes and dimensions and are of tremendous interest for the nanofabrication of sub-30 $\mathrm{nm}$ ordered structures, for example, photonic crystals [1], membranes with dense arrays of pores [2,3], lithographic templates for patterning applications [4], and complex, threedimensional structures [5]. The phase behavior and domain dimensions of symmetric ternary blends is determined by the volume fraction of total homopolymer in the blend, $\phi_{H}$, and the ratio of the degree of polymerization of the homopolymers to that of the block copolymer, $\alpha$. These materials in the bulk have been characterized as a function of $\phi_{H}$ and $\alpha$ using small angle neutron scattering (SANS) [6-15], small angle $x$-ray scattering [15-17], transmission electron microscopy $[7,9,16]$, rheology $[8,18,19]$, light scattering [9,10,20], and theoretical approaches [21-23]. Below the order-disorder transition (ODT) temperature, symmetric ternary blends form lamellae at low $\phi_{H}$ and macrophase separated morphologies at high $\phi_{H}$. Mean field theory predicts that these phases should be separated by an unbinding transition (UT) [21]; however, it has been observed experimentally that concentration fluctuations induce the formation of a bicontinuous microemulsion over a range of $\phi_{H}$ that obscures the UT [7,8,10,13,16,17]. Despite these considerable theoretical and experimental efforts, the behavior of these materials is not fully understood even in bulk systems.

Here we investigate the phase behavior of symmetric block copolymer and homopolymer blends in $\sim 40 \mathrm{~nm}$ thin films on neutral surfaces and observe transitions similar to those in the bulk. Blend compositions in the lamellar region of the phase diagram are directed to assemble on chemically striped surfaces into ordered arrays with longrange perfection. Interestingly, thin films of blends with compositions in the microemulsion region of the diagram on striped surfaces are shown to either form lamellar
PACS numbers: 61.25.Hq, 64.60.Fr, 64.70.Nd, 81.16.Dn

phases or phase separate as a function of the pattern periodicity, and the behavior of these blends is discussed with respect to concentration fluctuations and confinement.

The examined ternary blends consisted of a symmetric poly(styrene- $b$-methyl methacrylate) copolymer (PS- $b$-PMMA, $N_{\mathrm{BC}}=1020$ ) and equal volume fractions of polystyrene (PS) and poly(methyl methacrylate) (PMMA) homopolymers. The blends had compositions with $0 \leq \phi_{H} \leq 1.0$ and $\alpha=0.4$. Films 40 to $45 \mathrm{~nm}$ in thickness were deposited on silicon substrates uniformly treated with neutral-wetting random copolymer brushes, PS- $r$-PMMA with 58 vol $\%$ PS, to induce perpendicularly oriented domains throughout the film $[24,25]$. The films were annealed for $7 \mathrm{~d}$ at $190{ }^{\circ} \mathrm{C}$ and characterized by topdown scanning electron microscopy (SEM).

The phase behavior of symmetric ternary blend thin films on neutral surfaces is shown in the SEM images of Fig. 1 [26]. In thin films the blends undergo morphological transitions with increasing $\phi_{H}$ from lamellae to microemulsions to macrophase separated structures. Lamellae were formed for blends with $\phi_{H} \leq 0.4$ and the microemulsion morphology first arose at a $\phi_{H}$ between 0.5 and 0.6. Ternary blends with $\phi_{H} \geq 0.8$ formed macrophase separated morphologies.

Figure 2 presents a Fourier transform (FT) analysis of the SEM images in Fig. 1. The FT analysis plots the radially averaged Fourier intensity, $I$, as a function of the magnitude of the wave vector, $q$. Lamellar phase blends characterized in Fig. 2(a) exhibited first order peaks at $q^{*}=$ $2 \pi / L_{B, L}$ and higher order $(n)$ peaks at $q=n q^{*}$, where $L_{B, L}$ is the lamellar periodicity. The natural lamellar periods of the $\phi_{H}=0,0.2$, and 0.4 blends were calculated based on $q^{*}$ to be $L_{B, L}=50.7,56.6$, and $69.0 \mathrm{~nm}$, respectively, and in general $L_{B, L}=L_{O}\left(1-\phi_{H}\right)^{-0.74}$ for blends with $\alpha=$ 0.4 where $L_{O}$ is the lamellar periodicity of the pure diblock copolymer. This scaling of lamellar blend periodicity with $\phi_{H}$ is consistent with that found by Torikai et al. [15]. 

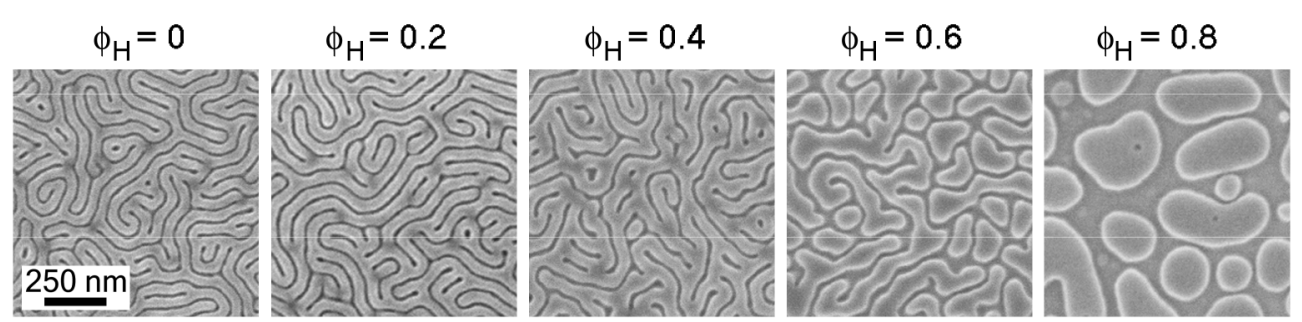

$\phi_{\mathrm{H}}=1.0$

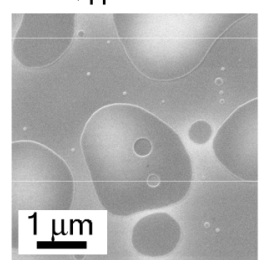

FIG. 1. Phase behavior of PS/PMMA ternary blends in thin films as a function of $\phi_{H}$. The SEM images of the $\phi_{H}=0$ to 0.8 blends are $1 \times 1 \mu \mathrm{m}^{2}$, and the light and dark regions are the PS and PMMA domains, respectively.

According to interpretations of SANS spectra of ternary blend systems in the bulk $[7,8]$, the onset of the microemulsion morphology can be determined by the disappearance of the second and higher order peaks in the FT analysis (see Fig. 2). Therefore, the FT data in Fig. 2 indicate that the lamellar to microemulsion transition occurred for the PS/PMMA blends at a composition of $0.5<$ $\phi_{H}<0.6$. A broad first order peak dominated the $I$ versus $q$ data for the $\phi_{H}=0.6$ blend, but both a first order and a weak second order peak were observed for the $\phi_{H}=0.5$ blend. In addition, Fig. 2(b) demonstrates that the $\phi_{H}=$ 0.6 blend had a FT peak with the Teubner-Strey (T-S) form typical of microemulsions [28]. The T-S form does well to describe the experimental structure data at low $q$, but the shoulder present at $q \sim 2 q^{*}$ likely resulted from the remnants of lamellae observed in the SEM image of Fig. 1 [8]. The peak of the T-S fit corresponded to a characteristic dimension for the $\phi_{H}=0.6$ microemulsion morphology of $L_{B, \mu E}=101 \mathrm{~nm}$.

The homopolymer-rich blends with $\phi_{H}=0.8$ and 1.0 macrophase separated into micron sized droplets of PS in a PMMA matrix. The FT peaks disappeared altogether and Fig. 2(c) verifies that the $I$ versus $q$ curves for the $\phi_{H}=$ 0.8 and 1.0 blends decayed with the $q^{-2}$ dependence of the Ornstein-Zernike form typical of two-phase morphologies [8,29].

The lamellar, microemulsion, and macrophase separated morphologies observed in two-dimensional thin films are similar to those in the three-dimensional bulk cases, but the transitions between phases as a function of $\phi_{H}$ differ between the bulk and confined systems. The microemulsion morphology observed here in the thin films on neutral surfaces first arose at $\phi_{H} \approx 0.60$. Mean field calculations with the random phase approximation have been used to predict a Lifshitz point, and therefore an assumed UT, at $\phi_{H} \approx\left(1+2 \alpha^{2}\right)^{-1}=0.78$ for $\alpha=0.4$ [21]. It is unclear, however, if this difference in transition composition can be attributed to confinement effects alone. Prior experimental and theoretical results have focused on blends in the weak segregation limit $(\chi N<15)$, whereas the PS and PMMA blends examined here are in the intermediate segregation regime $(\chi N=36.7)$. Even in bulk systems it remains unknown how the UT and the microemulsion region in the phase diagram extend to the intermediate or strong segregation limits.
The domain structures of diblock copolymer thin films can be directed to assemble on chemically nanopatterned surfaces with perfection and registration over arbitrarily large areas [4,24,30,31]. Ternary blends may hold some advantages for directed assembly over pure block copolymers because (1) the periodicity of the lamellar blends can be matched to the desired pattern dimensions simply by tuning the blend composition $[7,15,16,32]$ and (2) homopolymer redistribution within the blend domains facilitates the formation of nonregular geometries such as sharp bends [4]. We have therefore characterized the behavior of symmetric ternary blends on chemically striped surfaces
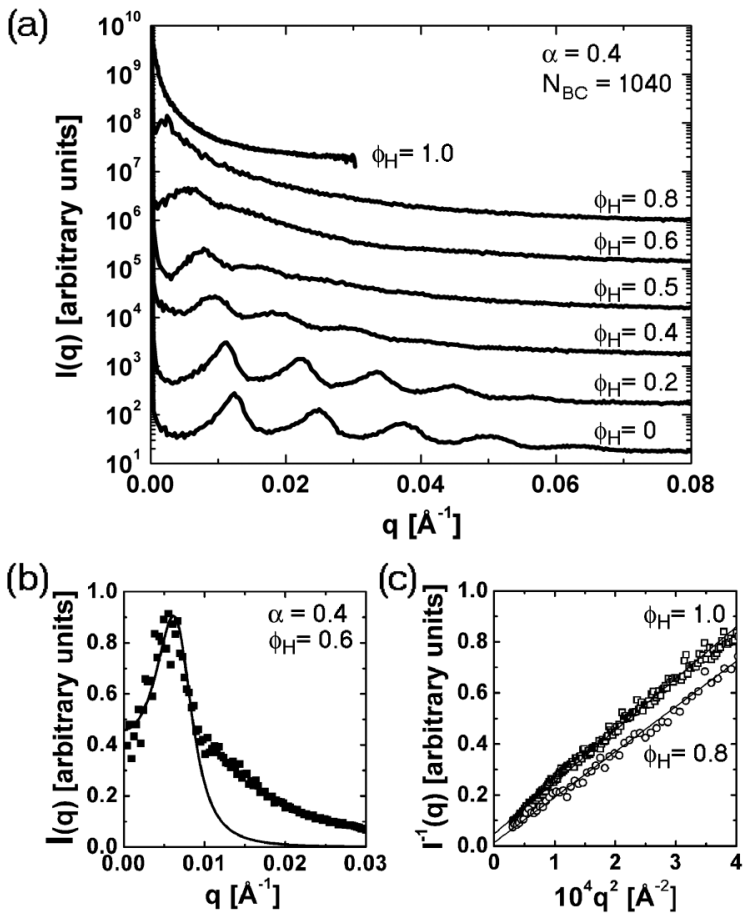

FIG. 2. FT analysis of the morphologies of the PS/PMMA ternary blends in thin films. (a) SEM images of the blends with $0 \leq \phi_{H} \leq 1.0$ (cf. Fig. 1) were converted to black/white images and used to calculate the radially averaged FTs of $I$ versus $q$. The individual curves have been offset in $I$ for clarity. (b) The FT data for the $\phi_{H}=0.6$ blend (filled squares) were fit by the Teubner-Strey expression (solid line) using only the $q<$ $0.01 \AA^{-1}$ data. (c) Ornstein-Zernike fits (solid lines) of the $\phi_{H}=0.8$ (open circles) and 1.0 (open squares) blends are linear in $I^{-1}$ versus $q^{2}$. 
with periodicities, $L_{S}$, from 45 to $110 \mathrm{~nm}$. These chemically patterned surfaces had alternating stripes of PS brush and oxygenated brush that were wet by the PS and PMMA domains of the blend, respectively. The fabrication of such surfaces has been detailed elsewhere $[4,31,33]$.

Figure 3 shows SEM images of the directed assembly of lamellar and microemulsion-forming blends on chemically patterned surfaces. Compositions in the lamellar region of the phase diagram $\left(\phi_{H}=0\right.$ to 0.4$)$ assembled into defect-free lamellae that were oriented and registered on underlying chemical surface patterns with $L_{S}$ between $0.87( \pm 0.05) L_{B, L}$ and $1.10( \pm 0.05) L_{B, L}$ [see Fig. 3(a)]. The behavior of blends in the lamellar region of the phase diagram $\left(\phi_{H} \leq 0.4\right)$ on chemical surface patterns with commensurate dimensions is similar to that of pure diblock copolymers on identical patterned surfaces as studied by experiment [24,30,31] and theory [34-37]. Favorable interfacial interactions between the polymer and the chemical surface pattern are sufficient when $0.90 \leq L_{S} / L_{B, L} \leq 1.10$ to yield equilibrated structures with the polymer chains and lamellar domains stretched or compressed to match $L_{S}$ [31].

Blends that formed microemulsions on neutral surfaces assembled into lamellar phases as shown in Fig. 3(b) when $L_{S}$ was equal to or slightly greater than $L_{B, \mu E}$. One possible explanation for the phase transition of the $\phi_{H}=0.6$ blend on surface patterns with commensurate dimension is that the substrate pattern pins the positions of the PS-PMMA interfaces and suppresses concentration fluctuations, thereby inhibiting the formation of a microemulsion morphology. In this scenario the UT occurs at the same homopolymer concentration, but the range of $\phi_{H}$ over which the microemulsion arises is reduced. Self-assembled systems on surface patterns may therefore provide excellent experimental comparisons to predictions from self-consistent field theory approaches which do not include fluctuations. A second explanation for the observed microemulsion behavior could be that the entire phase diagram for the ternary blends is modified by the presence of patterned surfaces. The UT and associated microemulsion morphology could shift to blends with higher concentrations of homopolymers, or alternatively, new morphologies could be formed $[5,19]$. For example, we have previously demonstrated that a blend that forms lamellae on neutral surfaces $\left(\phi_{H}=0.4\right)$ can assemble into unique bicontinuous structures on chemically patterned surfaces with a mismatched geometry [5]. Steady shear fields also have been used to induce the formation of new morphologies, e.g., distorted microemulsions and three-phase structures, from bulk polymeric microemulsions [19].

The $\phi_{H}=0.6$ microemulsion blend on patterned surfaces with $L_{S}<L_{B, \mu E}$, however, underwent a pattern induced separation into two coexisting phases, a homopolymer-rich macrophase separated morphology and a lamellar phase with a periodicity that matched $L_{S}$ (see Fig. 4). The blend assembly process began by excluding homopolymer in order to achieve directed lamellae with the imposed $L_{S}$ dimension. Homopolymer that was expelled from the lamellar structures aggregated in regions that then became enriched in homopolymer and that macrophase separated. Wetting layers at the near surface region are expected to perfectly follow the chemical pattern underneath these homopolymer enriched drops and are similar to those formed by binary homopolymer blends on heterogeneous surfaces [30]. The exact composition of the polymer layer that wets the surface pattern and the persistence length of the wetting layer into the film is unknown. Approximately 10 to $15 \mathrm{~nm}$ of topography between the lamellar and macrophase separated regions was measured by atomic force microscopy, which differs from the nearly uniform film thicknesses observed for the lamellar and microemulsion cases on homogeneous neutral surfaces and perfectly directed lamellae on chemical surface patterns.

(a) Lamellar Phase $\left(\alpha=0.4, \phi_{H}=0,0.2\right.$, or 0.4$)$
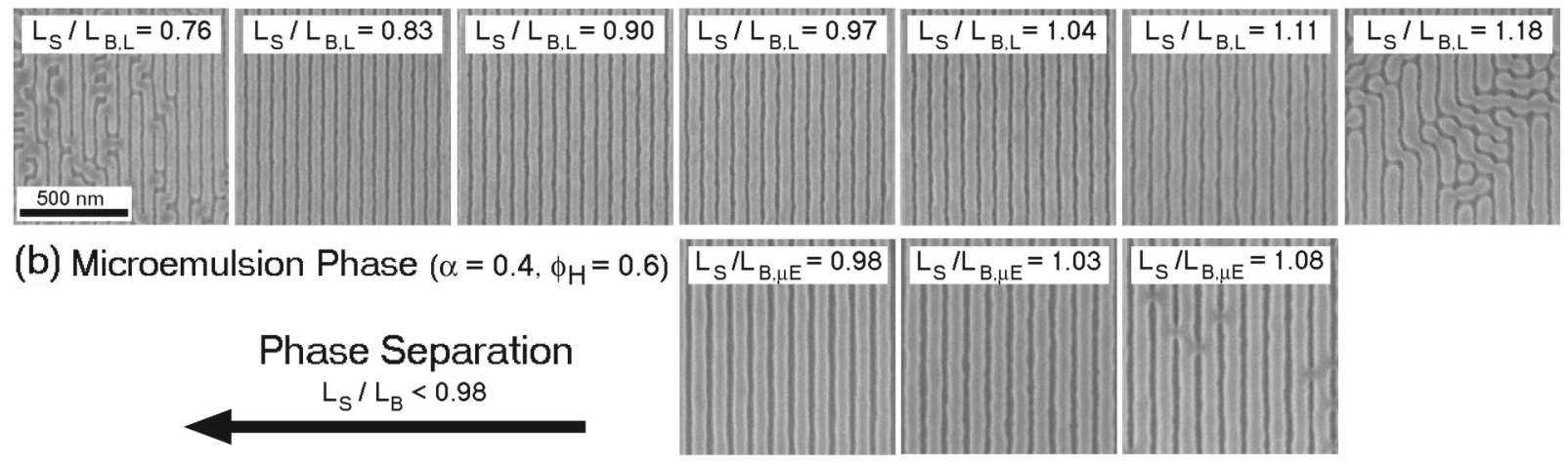

FIG. 3. Top-down SEM images of the directed assembly of PS/PMMA blends on chemically striped surfaces of periodicity $L_{S}$. (a) Lamellar-forming blends (here $\phi_{H}=0.4$ ) assemble with perfection over a wide range of $L_{S} / L_{B, L}$. (b) Microemulsion-forming blends (here $\phi_{H}=0.6$ ) assemble into lamellar structures on chemical surface patterns when $L_{S} / L_{B, \mu E} \approx 1$.0. Surface patterns with $L_{S} / L_{B, \mu E}<0.98$ induce a phase separation behavior. In all cases the SEM images shown are representative of much larger areas. 


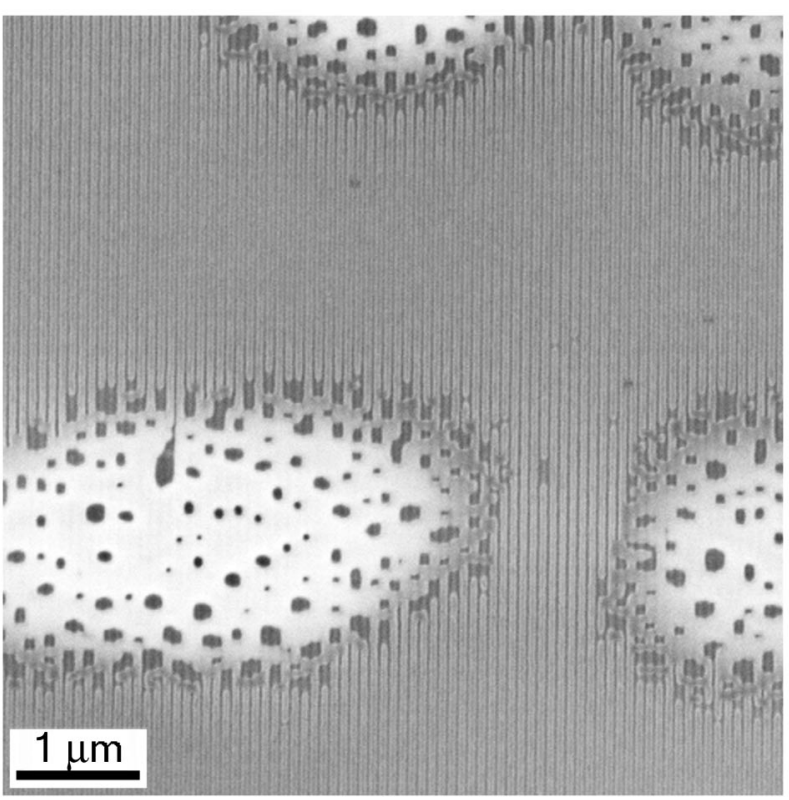

FIG. 4. Phase separation behavior of a microemulsion forming $\phi_{H}=0.6$ PS/PMMA blend on chemically patterned surfaces with $L_{S}=80 \mathrm{~nm}<L_{B, \mu E}=101 \mathrm{~nm}$. The morphology consists of coexisting regions of defect-free lamellae with $L_{B, L}=80 \mathrm{~nm}$ (gray stripes) and macrophase separated domains (white spots).

The behavior of blends that macroscopically phase separate has not been studied here on patterned surfaces, but Boltau et al. [38] and Karim et al. [39] showed that binary homopolymer blends $\left(\phi_{H}=1.0\right)$ can be directed to assemble when the characteristic dimension of the phase separated structures (of order microns) matches $L_{S}$.

The phase behavior of self-assembling symmetric ternary blends in thin films and on chemically patterned surfaces is not only of fundamental interest, but also of technological importance for nanolithography. Control over the molecular parameters of the blends provides a simple approach for achieving the variety of dimensions required for the patterning of complex devices. Redistribution of homopolymer within the blend domains facilitates the defect-free fabrication of the arbitrary geometries required in many lithographic applications. Directed assembly of neighboring structures with different dimensions and geometries on the same subfield, a critical requirement for nanomanufacturing [40], may be further enabled by purposefully designing into the pattern layout polymer reservoirs, which could act as homopolymer sources or sinks for the surrounding patterned areas.

We thank Dr. Marcus Müller for many insightful discussions. Support was provided by the Semiconductor Research Corporation (SRC) (No. 2005-OC-985) and the NSF UW Nanoscale Science and Engineering Center (No. DMR-0425880). This work used the facilities and staff at the UW Synchrotron Radiation Center (NSF No. DMR-0084402) and the Swiss Light Source at the Paul Scherrer Institut. M.P. S. acknowledges the SRC for financial support.
*To whom correspondence should be addressed. Electronic address: nealey@engr.wisc.edu

[1] A. Urbas et al., Adv. Mater. 12, 812 (2000).

[2] U. Y. Jeong et al., Adv. Mater. 14, 274 (2002).

[3] U. Y. Jeong et al., Macromolecules 36, 10126 (2003).

[4] M. P. Stoykovich et al., Science 308, 1442 (2005).

[5] K. C. Daoulas et al., Phys. Rev. Lett. 96, 036104 (2006).

[6] F. S. Bates et al., Phys. Rev. Lett. 75, 4429 (1995).

[7] F. S. Bates et al., Phys. Rev. Lett. 79, 849 (1997).

[8] M. A. Hillmyer et al., J. Phys. Chem. B 103, 4814 (1999).

[9] T. L. Morkved et al., Faraday Discuss. 112, 335 (1999).

[10] T. L. Morkved et al., J. Chem. Phys. 114, 7247 (2001).

[11] P. Stepanek et al., Physica A (Amsterdam) 314, 411 (2002).

[12] D. Schwahn et al., Phys. Rev. Lett. 82, 5056 (1999).

[13] D. Schwahn et al., J. Chem. Phys. 112, 5454 (2000).

[14] K. Mortensen et al., J. Appl. Crystallogr. 33, 686 (2000).

[15] N. Torikai et al., Macromolecules 30, 5698 (1997).

[16] L. Corvazier et al., J. Mater. Chem. 11, 2864 (2001).

[17] L. Messe, L. Corvazier, and A. J. Ryan, Polymer 44, 7397 (2003).

[18] G. Floudas et al., J. Chem. Phys. 106, 3318 (1997).

[19] K. Krishnan et al., Phys. Rev. Lett. 87, 098301 (2001).

[20] P. Stepanek et al., Macromol. Symp. 149, 107 (2000).

[21] D. Broseta and G. H. Fredrickson, J. Chem. Phys. 93, 2927 (1990).

[22] D. Duchs et al., Macromolecules 36, 9237 (2003).

[23] D. Duchs and F. Schmid, J. Chem. Phys. 121, 2798 (2004).

[24] S. O. Kim et al., Nature (London) 424, 411 (2003).

[25] P. Mansky et al., Science 275, 1458 (1997).

[26] Top-down SEM images describe the morphologies throughout the $\sim 42 \mathrm{~nm}$ films because (1) perpendicular orientations of lamellae have been achieved on neutral surfaces through much thicker films [27] and (2) the free surface energies for the PS and PMMA components are nearly equal at these processing conditions such that the top surface can virtually act as a neutral interface.

[27] P. Mansky et al., Macromolecules 30, 6810 (1997).

[28] M. Teubner and R. Strey, J. Chem. Phys. 87, 3195 (1987).

[29] J.S. Higgins and H.C. Benoit, Polymers and Neutron Scattering (Oxford University, New York, 1994).

[30] L. Rockford et al., Phys. Rev. Lett. 82, 2602 (1999).

[31] E. W. Edwards et al., Adv. Mater. 16, 1315 (2004).

[32] K. A. Orso and P.F. Green, Macromolecules 32, 1087 (1999).

[33] H. H. Solak et al., Microelectron. Eng. 67, 56 (2003).

[34] K. L. Huang and A.C. Balazs, Phys. Rev. Lett. 66, 620 (1991).

[35] G. G. Pereira and D. R. M. Williams, Phys. Rev. Lett. 80, 2849 (1998).

[36] H. Chen and A. Chakrabarti, J. Chem. Phys. 108, 6897 (1998).

[37] Q. Wang et al., J. Chem. Phys. 112, 9996 (2000).

[38] M. Boltau et al., Nature (London) 391, 877 (1998).

[39] A. Karim et al., Phys. Rev. E 57, R6273 (1998).

[40] D. J.C. Herr, in Future Fab International, edited by B. Dustrud (Montgomery Research Incorporated, San Francisco, 2006), Vol. 20. 